\section{Atenção à saúde dos adolescentes: percepção dos médicos e enfermeiros das equipes da saúde da família}

\author{
Healthcare for adolescents: perceptions \\ by physicians and nurses in family health teams
}

Rosângela Aparecida Pimenta Ferrari ${ }^{1}$
Zuleika Thomson ${ }^{1}$
Regina Melchior ${ }^{1}$
Abstract

This descriptive qualitative study was conducted in basic healthcare units to analyze the perceptions of physicians and nurses from family health teams concerning healthcare for adolescents, using content analysis with an emphasis on thematic analysis. The discourse of these health professionals showed that care exists for adolescents in the family health service, but that: it is not systematized because of other priorities; adolescents fail to use the service; the health professionals feel unprepared to draw adolescents to the service and treat them; and in order to implement a healthcare program for adolescents in the family health strategy it would be necessary to reorganize the service to train the existing team members and hire other professionals. Thus, the interviewees value differentiated care for adolescents, and even while recognizing their limitations and reporting not having been trained, they conduct joint actions with other areas beyond health, demonstrating that they transcend the limits of the health service and seek new ways of treating adolescents holistically.

Adolescent; Teen Health; Primary Health Care; Family Health Program

\section{Introdução}

No Brasil, as políticas públicas para adolescentes, criadas e regulamentadas na década de 80 , se desenvolvem de forma fragmentada e desarticulada, "cada setor do governo desenvolve suas politicas, estratégias e ações isoladamente (...) não representando um trabalho intersetorial expressivo dirigido à integralidade da atenção ao adolescente" 1 (p. 731).

Em face dessa realidade, em uma outra perspectiva, a política de saúde que pode mudar a situação atual dos adolescentes no país é a Estratégia da Saúde da Família (ESF), por se aproximar mais das condições socioculturais e assim cumprir os princípios que norteiam o SUS 2.

Nesse sentido, o estudo teve como objetivo analisar a percepção dos médicos e enfermeiros sobre a atenção à saúde do adolescente na ESF.

\section{Metodologia}

Investigação qualitativa descritiva, desenvolvida com médicos e enfermeiros das equipes da saúde da família das Unidades Básicas de Saúde (UBS) do Município de Londrina, Paraná, Brasil, nos meses de agosto a outubro do ano de 2003.

A coleta de dados foi realizada por meio de um formulário composto por questões fechadas e uma questão aberta, para quem desejas- 
se discursar a próprio punho, no local de trabalho, sobre a temática de pesquisa.

A partir do registro dos profissionais na questão aberta do formulário foi realizada a análise de conteúdo, especificamente a análise temática que "consiste em descobrir os núcleos de sentido que compõem uma comunicação cuja presença ou freqüência signifiquem alguma coisa para o objetivo analítico visado" 3 (p. 210), e o tema "é a unidade de significação que se liberta naturalmente de um texto analisado segundo critérios relativos à teoria que serve de guia à leitura" 4 (p. 105).

Para proceder à análise temática foi organizado e estruturado o conteúdo da questão aberta seguindo as fases seqüenciais propostas por Minayo 3: a pré-análise, a exploração do material e o tratamento dos resultados.

A pesquisa foi aprovada pelo Comitê de Ética em Pesquisa da Universidade Estadual de Londrina (parecer CEP 176/02 em 11 de dezembro de 2001).

\section{Resultados e discussão}

A questão aberta do formulário foi preenchida por 47 profissionais resultando na categorização de cinco temas: (i) os serviços de saúde e o adolescente: pouca oferta ou baixa procura?; (ii) ações de atenção à saúde do adolescente; (iii) necessidade de formação/capacitação sobre adolescência; (iv) multidisciplinaridade e intersetorialidade e; (v) necessidade de implantação de um programa específico para adolescentes.

\section{Os serviços de saúde e o adolescente: pouca oferta ou baixa procura?}

Esse tema revela, por meio dos discursos dos médicos e enfermeiros, que o adolescente recebe atendimento no serviço, mas não de forma sistematizada e sim de acordo com a demanda da unidade, pois existem outras prioridades, adiando assim a organização de trabalho com esse grupo etário: “...não desenvolvo ações de saúde sistematizadas, devido à alta demanda de outros problemas na UBS..." (médico 4); “...estamos implantando algumas outras ações: grupo de diabetes, hipertensos, asmáticos, ainda não nos foi possível implementar algo específico para adolescentes" (médico 13).

Enquanto que em algumas unidades a demanda do atendimento ao adolescente é suprimida, em outras é crescente, principalmente entre as garotas, mas sente-se dificuldades em conquistá-lo para que procure o serviço espon- taneamente: "o número de meninas adolescentes em procura de exames laboratoriais para confirmar gestação tem aumentado, procura para coleta de material para preventivo de câncer uterino e outros (...) já adolescentes meninos a procura é baixa, mais um ponto para reflexão" (enfermeiro 30); "temos muita dificuldade para trabalhar com os adolescentes, visto que, os mesmos raramente participam das atividades propostas..." (enfermeiro 15).

Para viabilizar o acesso do adolescente no serviço de saúde "é fundamental que a atividade seja interessante e escolhida por eles, caso contrário, mesmo quando profusamente oferecida, o comparecimento tenderá a ser muito baixo" 5 (p. 179).

É difícil afirmar se a relativa ausência dos adolescentes nos serviços de saúde se deve à pouca oferta de ações voltadas para eles ou à baixa procura dos mesmos, uma vez que estes dois fatores estão interligados e se referem à forma como o serviço de saúde está estruturado atualmente no país e, também existir poucos estudos sobre esse enfoque.

\section{Ações de atenção à saúde dos adolescentes}

Quanto à prática de ações de prevenção e promoção da saúde desenvolvidas pelos médicos e enfermeiros no serviço para os adolescentes, alguns dos discursos referem que estas geralmente são individuais, mas quando desenvolvidas em grupo são realizadas na comunidade e na escola: "a prevenção em minha USF é realizada de uma forma individualizada. Já iniciamos o trabalho na escola (...) estamos criando vínculo com eles através do contato na comunidade escolar, estamos com proposta de paralelo a este trabalho desenvolver um grupo na comunidade como bate-papo do adolescente" (enfermeiro 21).

Pode-se observar que existem iniciativas de trabalho com adolescentes e mesmo que isoladas são importantes, pois "a partir do momento em que os profissionais da estratégia reorganizam suas atividades, as necessidades e ações de saúde irão se corresponder, e não só os problemas de saúde determinarão as ações como também o modo de organização do serviço corresponderá às necessidades de saúde, contemplando a dupla dimensão, a individual e a coletiva, do processo de saúde doença ampliando o campo da atenção e do cuidado" 6 (p. 17).

Nesse sentido, ao ampliar o campo de atenção e cuidado poder-se-á contemplar o adolescente na agenda do serviço de saúde, favorecendo o aumento da procura e conseqüente- 
mente o aumento da oferta de serviços nas unidades básicas de saúde e respectivas áreas de abrangência.

\section{Necessidade de formação/ capacitação sobre adolescência}

Antes mesmo de desenvolver atividades com grupos de adolescentes, os médicos e enfermeiros referem a necessidade da equipe participar de cursos de formação/capacitação sobre essa fase da vida, visto que alguns dos discursos apontam pouco conhecimento e dificuldade para lidar com esse grupo “...pessoalmente, por falta de preparo, sinto alguma dificuldade para manter contato com essa faixa etária" (médico 19); “...necessitamos de capacitação para podermos atendê-los melhor, visto que a linguagem, a forma de abordar, os vínculos e a confiança têm que atender as necessidades dos adolescentes" (enfermeiro 20).

Além das dificuldades e pouco embasamento sobre a adolescência, referidos pelos profissionais, alguns discursos emitem também julgamentos, considerando-os deseducados e permissivos: “...deveríamos orientar o sexo no casamento e desaconselhar essa permissividade atual” (médico 10); “...o profissional do PSF pode contribuir para a diminuição de gravidez precoce e DST, porém a formação, ou diria não educação dos adolescentes fazem com que haja um desrespeito muito grande aos professores ou mesmo palestrantes" (médico 14).

Tais julgamentos, geralmente são advindos do pouco e até nenhum conhecimento dos profissionais sobre a fase da adolescência, conseqüentemente criando barreiras na relação e interação com essa clientela.

Alguns estudos concluíram que uma das dificuldades dos profissionais de saúde no trabalho com adolescentes era proveniente do ensino de graduação, por não contemplar o tema adolescência e sexualidade humana na grade curricular 7,8,9,10,11,12,13 e, quando contempladas, as contribuições estão voltadas para o controle normativo e tecnológico da sexualidade e para o processo reprodutivo, com pouco ou até nenhum trabalho que englobe gênero, classe e etnia $11,13,14$.

Mas, enquanto que a formação acadêmica das áreas afins não tem dado conta do ensino da adolescência e sexualidade humana no enfoque além do biológico, é imprescindível maiores investimentos na educação permanente dos profissionais nos serviços de saúde e assim viabilizar a assistência integral à saúde do adolescente.

\section{Multidisciplinaridade e intersetorialidade}

Para abordar a adolescência na complexidade de conhecimentos psicossocial-cultural e político, é fundamental desenvolver trabalhos multidisciplinar e interdisciplinar, e alguns dos discursos dos profissionais referem que "seria interessante que as atividades fossem desenvolvidas por uma equipe multidisciplinar como assistente social, psicóloga, médicos, enfermeiros etc., pois não temos como desenvolver e resolver os problemas apresentados sem apoio dos outros profissionais, e isto sobrecarrega os profissionais médicos e enfermeiros" (enfermeiro 1).

Atuar multiprofissionalmente exige um trabalho em equipe com interação e troca nos campos de competência e abre a possibilidade de cada um usar todo o seu potencial criativo na relação com o usuário, para juntos realizarem a produção do cuidado 15.

Para isso, será preciso ir além do atendimento clínico e buscar parcerias com outros setores a fim de obter melhor e maior efetividade nas ações de atenção à saúde do adolescente. Alguns dos discursos dos profissionais referem essa necessidade: "precisamos de uma ação que envolva a saúde, a educação e toda a comunidade" (enfermeiro 15); "para o atendimento ser mais efetivo deveria existir uma parceria entre a Secretaria de Saúde e Secretaria de Educação" (enfermeiro 24).

Um dos desafios da intersetorialidade é identificar objetivos comuns e buscar, mediante um núcleo estratégico de planejamento e definição, prioridades de ações. 12 Identificar objetivos comuns intersetorialmente e multidisciplinarmente não significa “...somar conhecimentos, mas transformá-los num outro, apropriado às necessidades específicas do trabalho com adolescentes" 5 (p. 144). E parece que somar não tem sido tarefa fácil na prática das instituições produtoras do cuidado à saúde da população.

\section{Necessidade de implantação de um programa específico para adolescentes}

Profissionais que trabalham em programas de atenção à saúde de adolescentes referem dificuldades no trato diário com esse grupo etário, pois para que se cumpra a agenda de atividades os esforços parecem ser em vão, causandolhes frustração, desmotivação, mal-estar e até mesmo irritação, pois geralmente não comparecem no serviço 16,17 .

Mesmo que alguns profissionais refiram dificuldades para desenvolver ações de atenção à saúde do adolescente e também captá-lo, neste estudo os discursos dos médicos e enfermei- 
ros apontam para a predisposição para implantar um programa na equipe da saúde da família, mas alertam para a necessidade da manutenção de apoio durante a sua realização: “...pela importância e pela atualidade do assunto, acredito que todas UBS deveriam ter um programa para adolescentes, como tantos outros já existentes" (médico 9); "vejo a necessidade de melhores serviços de referência para este grupo, incluindo serviço de apoio para as equipes de PSF" (enfermeiro 25).

\section{Resumo}

Trata-se de uma investigação qualitativa descritiva, desenvolvida nas Unidades Básicas de Saúde, para analisar a percepção dos médicos e enfermeiros das equipes da saúde da família sobre a atenção à saúde do adolescente, utilizando-se a Análise de Conteúdo com ênfase na Análise Temática. Os discursos dos profissionais revelaram que existe atendimento aos adolescentes no serviço, mas não é sistematizado por terem outras prioridades; que o adolescente não procura o serviço; se sentem despreparados para atender e captá-los e; para implantar um programa de atenção à saúde do adolescente na estratégia será necessário reorganizar o serviço para capacitar os profissionais das equipes e inserir outros profissionais. Pode-se concluir que os profissionais consideram importante o atendimento diferenciado aos adolescentes, reconhecem suas limitações e mesmo que refiram não terem sido capacitados já desenvolveram algumas ações conjuntas com outras áreas, além da saúde, demonstrando que transcendem os limites do serviço buscando novos caminhos para atender o adolescente na sua integralidade.

Adolescente; Saúde do Adolescente; Atenção Primária à Saúde; Programa Saúde da Família
Para tanto, é preciso rever a organização do trabalho nas equipes e consolidar uma política pública para "ampliar o acesso à rede de atenção básica de saúde, que por conseguinte deverá criar espaços de discussão e aprofundamento de questões formuladas pelos próprios adolescentes" 18 (p. 619), pois segundo alguns discursos “...o grande investimento a ser feito é o desenvolvimento de ações voltadas para esta parcela da população, pois estaremos contribuindo para uma melhoria da qualidade de vida de nossos futuros adultos e conseqüentemente modificando o perfil de saúde dos mesmos" (enfermeiro 22).

\section{Colaboradores}

Todas as autoras participaram das etapas de concepção e delineamento do estudo, de análise e interpretação dos resultados e de revisão crítica do trabalho.

\section{Agradecimentos}

Médicos e Enfermeiros da ESF, Dra. Leila Sollberger Jeolás, Dra. Zuleika Thomson e Dra. Regina Melchior, Bruna Camila F. da Silva, Fernanda Donádio Pitta e Giseli Plath. 


\section{Referências}

1. Rua MG. As políticas públicas e a juventude dos anos 90. In: Comissão Nacional de População e Desenvolvimento, organizador. Jovens acontecendo na trilha das políticas públicas. v. 2. Brasília: Ministério da Saúde/Comissão Nacional de População e Desenvolvimento; 1998; p. 731-49.

2. Secretaria de Assistência à Saúde, Ministério da Saúde. Saúde da família: uma estratégia para a reorientação do modelo assistencial. Brasília: Ministério da Saúde; 1998.

3. Minayo MCS. Pesquisa social: teoria, método e criatividade. Petrópolis: Editora Vozes; 2001.

4. Bardin L. Análise de conteúdo. Lisboa: Edições $70 ; 2002$.

5. Ayres JRCM. Adolescência e saúde coletiva: aspectos epistemológicos da abordagem programática. In: Schraiber LB, organizador. Programação em saúde hoje. São Paulo: Editora Hucitec; 1990; p. 139-82.

6. Peduzzi M. Equipe multiprofissional de saúde: conceito e tipologia. Rev Saúde Pública 2001; 35:103-9.

7. Ali Geha A. Sexualidade feminina: estudo sobre abordagem no serviço público [Monografia]. Londrina: Universidade Estadual de Londrina; 1998.

8. Peres F, Rosenburg CP. Desvelando a concepção de adolescência/ adolescente presente no discurso da Saúde Pública. Saúde Soc 1998; 7:53-86.

9. Torres GV. Atividades do enfermeiro na prevenção do HIV/Aids em Natal/RN: competências pedagógica e técnica [Tese de Doutorado]. Ribeirão Preto: Escola de Enfermagem, Universidade de São Paulo; 2000.

10. Gir E, Nogueira MS, Pelá NTR. Sexualidade humana na formação do enfermeiro. Rev Latinoam Enfermagem 2000; 8:33-40.
11. Carvalho AJL, Detsi MI. Sexualidade e adolescência: discurso e prática nas instituições de saúde. In: Vale JRA, organizador. Nas pegadas da juventude: estudos e pesquisas sobre adolescentes no Ceará. Fortaleza: Abreu Vale; 2001. p. 189-97.

12. Teixeira RA. O trabalho da enfermeira na saúde da família: potência de (re)construção do modelo assistencial e (re)criação do trabalho da enfermagem? [Dissertação de Mestrado]. Ribeirão Preto: Escola de Enfermagem, Universidade de São Paulo; 2002.

13. Loyola MA. Sexualidade e medicina: a revolução do século XX. Cad Saúde Pública 2003; 19:875-84.

14. Ayres JRCM, França Jr. I. Saúde do adolescente. In: Schriber LB, Nemes MIB, Gonçalves RBM, organizadores. Saúde do adulto: programa e ações na unidade básica. São Paulo: Editora Hucitec; 1996; p.67-85.

15. Franco TB, Merhy EE. PSF: contradições de um programa destinado à mudança do modelo tecnoassistencial. Campinas: Editora da Unicamp; 1999.

16. Abduch C. Grupos operativos com adolescentes. In: Schor N, Mota MSFT, Branco VC, organizadores. Cadernos juventude, saúde e desenvolvimento. Brasília: Ministério da Saúde, 1999. p. 289-300.

17. Ruzany MH, Andrade CLT, Esteves MAP, Pina MF, Szwarcwald CL. Avaliação das condições de atendimento do Programa de Saúde do Adolescente no Município do Rio de Janeiro. Cad Saúde Pública $2002 ; 18: 639-49$.

18. Jeolás LS, Ferrari RAP. Oficinas de prevenção em um serviço de saúde para adolescentes: espaço de reflexão e de conhecimento compartilhado. Ciênc Saúde Coletiva 2003; 8:611-20.

Recebido em 28/Jun/2005

Versão final reapresentada em 12/Mai/2006

Aprovado em 03/Jul/2006 